\title{
Rapid Fixation -Is it an Effective Method in the Diagnosis of Cytological Smears
}

\author{
Dr. Sonti Sulochana ${ }^{1 *}$, Dr. Jaswanth $\mathbf{M}^{2}$ \\ ${ }^{1}$ Professor of Pathology, Saveetha Medical College, Saveetha Nagar, Thandalam Chennai Bengaluru National Highway Thandalam, Chennai, Tamil \\ Nadu 602105, India \\ ${ }^{2}$ IInd year MBBS, Saveetha Medical College, Saveetha Nagar, Thandalam Chennai Bengaluru National Highway Thandalam, Chennai, Tamil Nadu \\ 602105, India
}

\author{
DOI: $10.36348 /$ sipm.2019.v04i11.006 \\ | Received: 09.11.2019 | Accepted: 17.11.2019| Published: 24.11.2019 \\ *Corresponding author: Sulochana Sonti
}

\section{Abstract}

\begin{abstract}
Aim: Rapid fixation -Is it an an effective method in the diagnosis of cytological smears. Objective: 1) Is it an accurate method in fine needle aspiration cytology. 2) Is it an accurate method in the diagnosis of various body fluid cytology. 3) To analyse the quality of background staining and cyto-morphology. 4) To compare the quality between rapid and routine fixation method. Methods: Smears are fixing in 95\% isopropyl alcohol and stained by hematoxyline and eosin and papaniecolou staining. Result: The cyto-morphology and background staining quality in rapid fixation and routine fixation were analysed and are almost equal to routine fixation method. Conclusion: By rapid fixation the adequacy of the smears can be screened and analysed quickly. So we can reduce the turnaround time (TAT) and more benefit for the patient

Keywords: Isopropyl alcohol, fluids, FNAC, centrifuge, H\&E and Pap staining.

Copyright @ 2019: This is an open-access article distributed under the terms of the Creative Commons Attribution license which permits unrestricted use, distribution, and reproduction in any medium for non-commercial use (NonCommercial, or CC-BY-NC) provided the original author and sources are credited.
\end{abstract}

\section{INTRODUCTION}

Cytology is the study of cells. It is a diagnostic stool that any changes in the individual cells are a reflection of the changes in the tissues from which they are derived. Exfoliative cytology is the study of cells .The material for cytological examination is obtained either from the cells that are spontaneously shed (exfoliated) from the epithelial surfaces of the body or which may be removed from such surfaces, membranes or cavities [1].

The most important features of exfoliative cytology are:

- This technique is applicable to organs which are easily accessible.

- The samples contain a wide variety of cells of various types obtained from different sources like inflammatory cells, macrophages, microorganisms, and material of extraneous origin.

- Due to ongoing process of exfoliation, the cellular constituents are at times poorly preserved.

- The most important advantage of exfoliative cytology is that multiple samples can be obtained from the same site.
The study of cytology is useful

- In the diagnosis of cancer in various organs, especially in the respiratory, urinary and female genital tract.

- Assessment of hormonal status in causes of sterility and endocrine disorders.

- Diagnosis of infections like fungal organisms in the smears of vaginal and respiratory cytology.

- Sex determination from the scrapings of buccal mucosa is used to see the Barr body in the nucleus of the cells.

The cytological fixatives are $95 \%$ ethyl alcohol, 95\% ethyl alcohol and 3\% glacial acetic acid and ether alcohol.

The cytological stains are

- Papanicolaous stain

- Romanowsky stains like Giemsa, Wrights and leishmans stain, and hematoxylin and eosin [1].

\section{MATERIALS AND METHODS}

The material collected from the cavities of body where exfoliated cells collect as a pool, eg 
Vaginal smears from posterior fornix or by directly scraping the epithelial membranes of certain areas of body cervical, buccal and vaginal scrapings and from secretions, body fluids and effusions like sputum, urine, pleural, ascitic, pericardial and CSF. committee.

This study is approved by Institutional ethical

\section{METHODOLOGY EQUIPMENTS}

- Needles; routinely 22-23 gauge needle used
- $\quad$ Syringes; $10 / 20 \mathrm{ml}$ syringe

- Pistol handle

- Sterile container; physiological saline or Hanks balanced solution

- $\quad$ Slides; Clean,dry and free of grease

- A $0.4 \mathrm{~mm}$ hemocytometer coverslip gives better control over smearing pressure and a more perfect spread

- Fixatives; 70-90\% ehanol/ 80\% isopropyl alcohol.

- Stains

- Microscopes
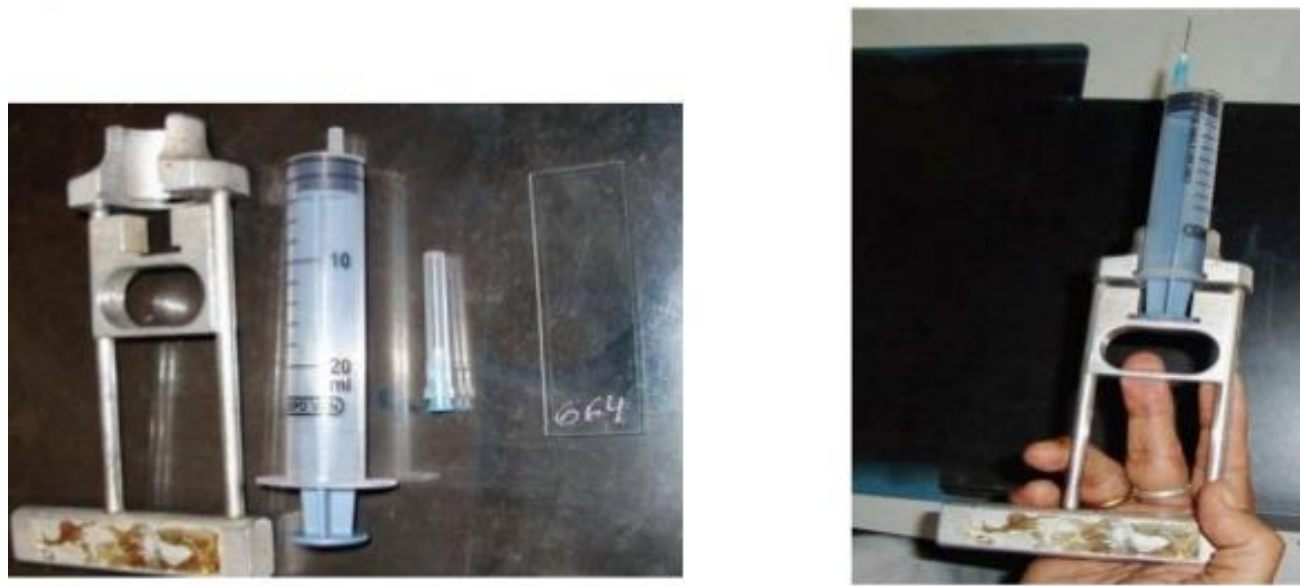

FINE NEEDLE ASPIRATION TECHNIQUE

Two techniques

1. FNAC with aspiration

2. FNAC without aspiration

\section{FNAC with aspiration}

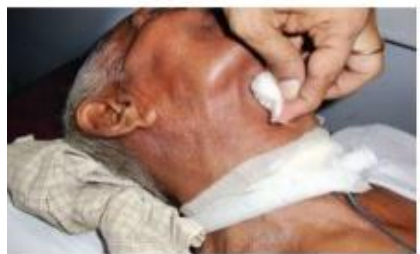

Site of FNAC should be cleaned by spirit swab

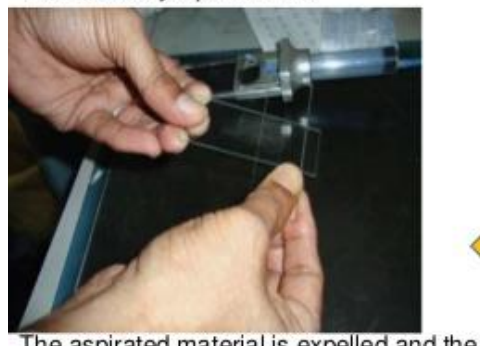

The aspirated material is expelled and the slide on the lower one

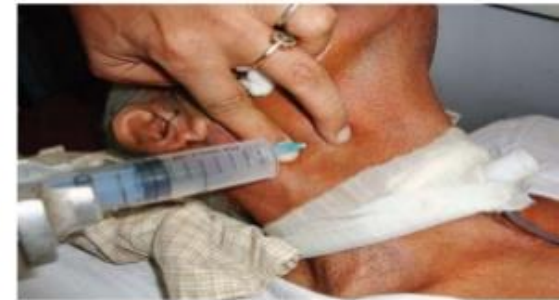

Needle is introduced in the swelling and is gently moving to and fro. Simultaneously negative suction is also created by withdrawing the piston
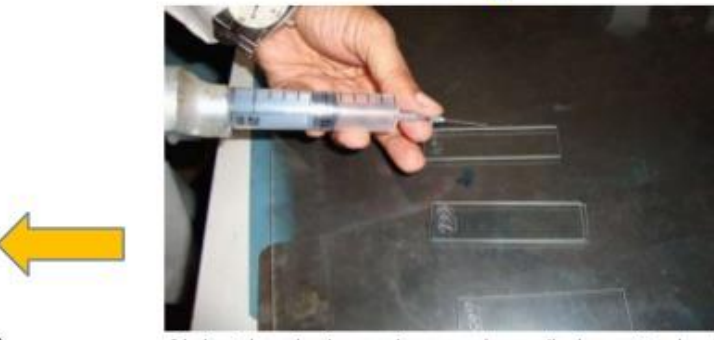

Air is taken in the syringe and needle is reattached 


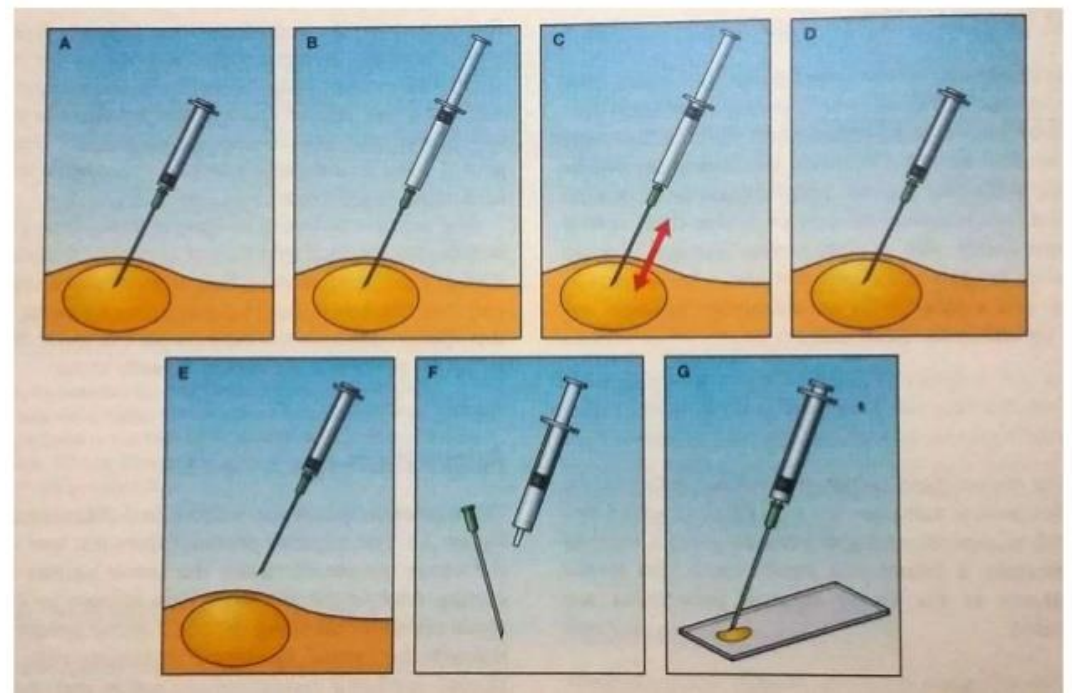

FNAC without aspiration

Introduced by Zajdela in 1987

Based on the observation that the capillary pressure in a fine needle is

Sufficient to keep the detached cells inside the lumen of the needle
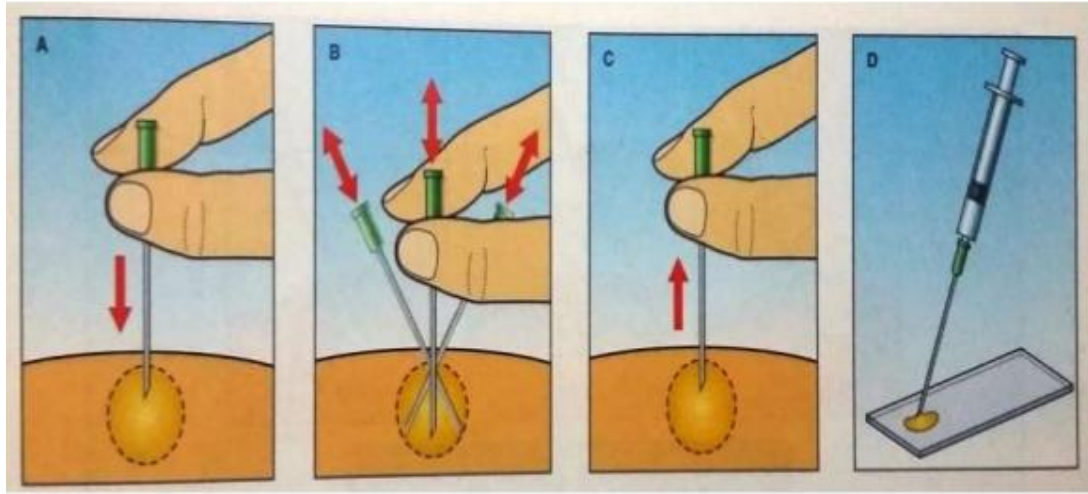

\section{BODY FLUIDS}
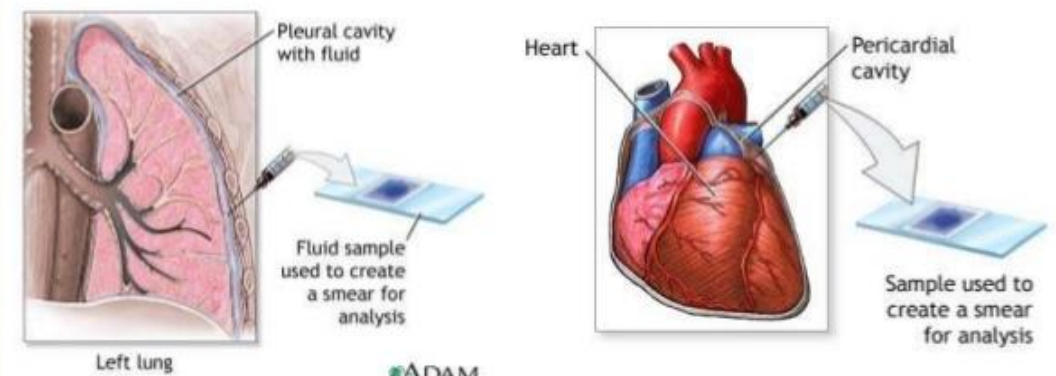

MAAM

\section{Pleural fluid}

\section{FIXATIVES}

Cytological fixatives are very much important to preserve intracellular structures or inclusion $[2,3]$.

The material for cytological examination obtained, In FNAC, after aspitration, smears are

prepared immediatetly lightly and evenly smeared on a clean, greasy free glass slide and smear is free from lumps and the slides are fixed in in $80 \%$ isopropyl alchol for 5 minutes and 20-30 minutes . 
The material from body cavities and effusions like urine, pleura, ascitic fluid, pericardial, peritoneal wash and gastric washings and Put these samples in clean air tight centrifuged tube. Rotate the tube at 1500 rounds per minute (RPM) for 5 minutes in Cytocentrifugation (REMI R-8C) [4]. Discard the supernatant fluid .Make multiple smears from the sediments on a clean glass slide. If RPM is more than $1500 / 10$ minutes may cause morphological distortion of cells, so care full attention should be given in this aspect. The slides are quickly placed in the fixative before drying of the material occurs. Slides should remain in the fixative for a minimum of 20-30 minutes or more prior to staining (There was no harm to keeping the slides for longer in the fixative). And the same smeared slides are fixed in $80 \%$ isopropyl alchol for 5 minutes and then taken out for routine staining.

\section{0\% ISO PROPYL ALCOHOL 90\% ISO PROPYL ALCOHOL \\ WATER 10\%}

Naturally colorless may contain color additives. This fixative can be used for about 7 days and it does not involve immediate preparation. It is the most commonly used fixative of choice.

After fixation the smears are then stained with routine haematoxylin and eosin stain.

Haematoxylin $5 \mathrm{~g}$

Absolute alcohol $50 \mathrm{ml}$

Alum $100 \mathrm{~g}$

Distilled water $1000 \mathrm{ml}$

Mercuric oxide $2.5 \mathrm{~g}$

EOSIN:

Eosin Y $16 \mathrm{~g}$

Potassium dichromate $8 \mathrm{~g}$

Picric acid $160 \mathrm{ml}$

95\% alcohol $160 \mathrm{ml}$

Distilled water $1280 \mathrm{ml}$

The smears are placed in haematoxylin for 3-5 minutes, then washed in running tap water for 2 seconds which then undergoes differentiation in alcohol followed by washing in tap water (bluing) and are then placed in eosin for $2 \mathrm{sec}$. The slides are then mounted and kept ready to pathologists for observation or analysis.

\section{PAPANICOLAOU STAINING}

- Named after Dr Geroge N Papanocolaou

- Polychrome staining reaction

- Display many variations of cellular morphology showing degree of cellular maturity and metabolic activity

\section{Principle}

- Hydration:

It prepares the cell sample for uptake of the nuclear staining

- Dehydration :

It prepares the cell sample for uptake of the counter stains

Dehydration and clearing solutions result in cellular transparency and prepare the cell sample for final steps

\section{Stains}

Nuclear staining; Haematoxylin

Two cytoplasmic counter staining

- Orange G-OG-6, OG-5 and OG-8 is an acidic dye which stains the keratin a bright intense orange

- Eosin Azure ; EA36, EA 50 and EA 65 including three stains

- Eosin Y

- Light green

- Bismarck brown Y Result

- Nuclei; Blue/black

- Cytoplasm(non keratinizing ); blue/green

- Keratinizing cells; pink/ orange

Table 3 Comparation of cytomorphological features in 5 minutes and more than 20-30 minutes by semiquantative scoring system

Table-1: Nuclearfeatures

\begin{tabular}{|l|l|l|l|l|}
\hline Fixation time & Score 0 & Score 1 & Score 2 & Score 3 \\
\hline 5 minutes & & & $\sqrt{ }$ & \\
\hline$>20-30$ minutes & & & & $\sqrt{ }$ \\
\hline
\end{tabular}

Table-2: Cytoplasmic features

\begin{tabular}{|l|l|l|l|l|}
\hline Fixation time & Score 0 & Score 1 & Score 2 & Score 3 \\
\hline 5 minutes & & & & $\sqrt{ }$ \\
\hline$>20-30$ minutes & & & & $\sqrt{ }$ \\
\hline
\end{tabular}

Table-3: Background features (mucoid and myxoid material, proteinaceous material, and RBCs)

\begin{tabular}{|l|l|l|l|l|}
\hline Fixation time & Score 0 & Score 1 & Score 2 & Score 3 \\
\hline 5 minutes & & & & $\sqrt{ }$ \\
\hline$>20-30$ minutes & & & & $\sqrt{ }$ \\
\hline
\end{tabular}

Score 0 - Unsatisfactory, Score 1 - Satisfactory Score, 2 - Good Score, 3 - Excellent 


\section{RESULT}

The cytomorphological staining quality of smears were analyzed by rapid and routine fixation. In this study, fixed the wet cell smears in $80 \%$ isopropyl alcohol for 5 minutes and routine staining with Haematoxylin and Eosin and papanicolaou staining was done. The representatitive rapid fixation and routine smears were compared for cellularity, background blood and cell debris, cell architecture, informative background (such as colloid, mucoid and stromal fragments), presence of cells in monolayer and clumps, and nuclear and cytoplasmic details by semiquantative scoring system (Table 1, $2 \& 3$ ).

The background staining quality and cell morphology like nucleus, cytoplasm features are studied (Fig 1 to 9). The cyto-morphology and background staining quality in rapid and routine fixation smears are similar to that of routine fixation there was very mild difference in nuclear features. In rapid fixation the nuclear staining are fair or good, but in fixation more than 30 minutes the nuclear staining is good/ very good. But there is no difficulty in reporting of smears by rapid fixation procedure.

\section{FIGURE WITH LEGENDS}

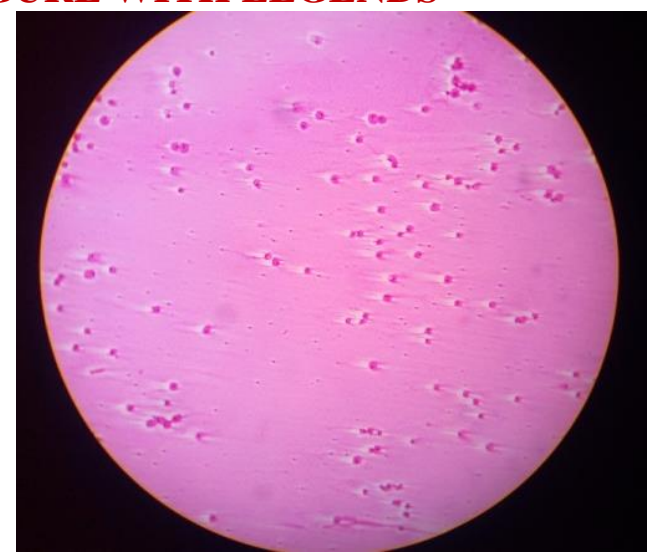

Fig-1a: 5 minutes fixation (H\&E 10X)

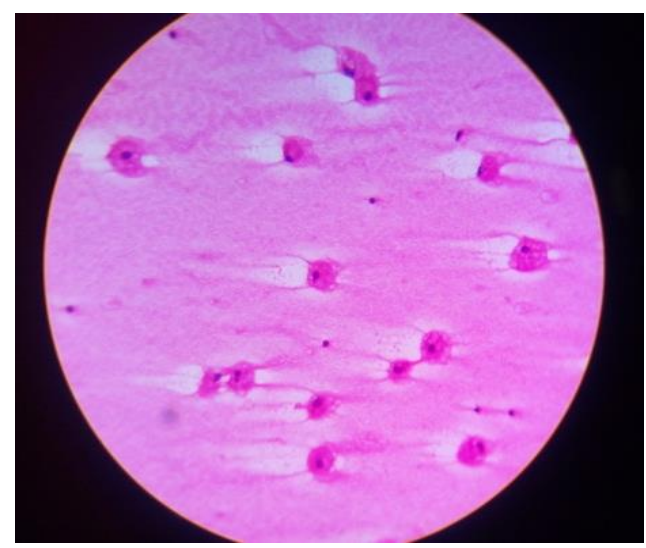

Fig-1b: 5 minutes fixation (H\&E 40X)

Nipple discharge shows foamy macrophages in a eosinophilic back ground.

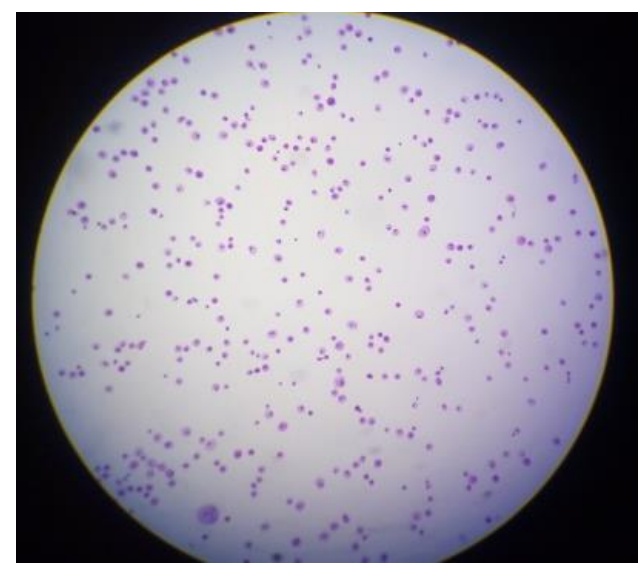

Fig-2a: >30 minutes fixation $(\mathrm{H} \& \mathrm{E} 10 \mathrm{X})$

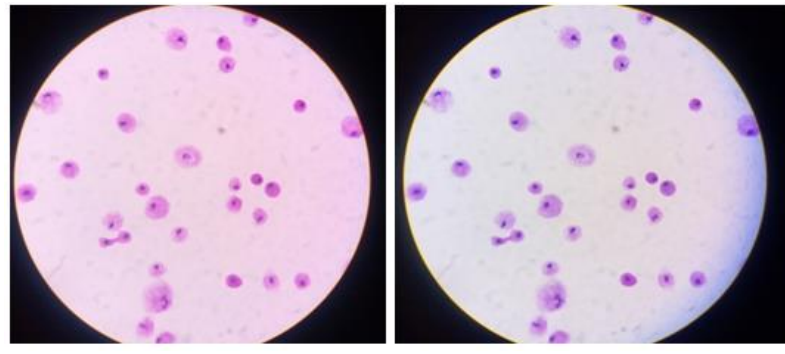

Fig-2b \& c: >30 minutes fixation (H\&E, \& Pap 40X

Nipple discharge shows foamy macrophages in an eosinophilic back ground.

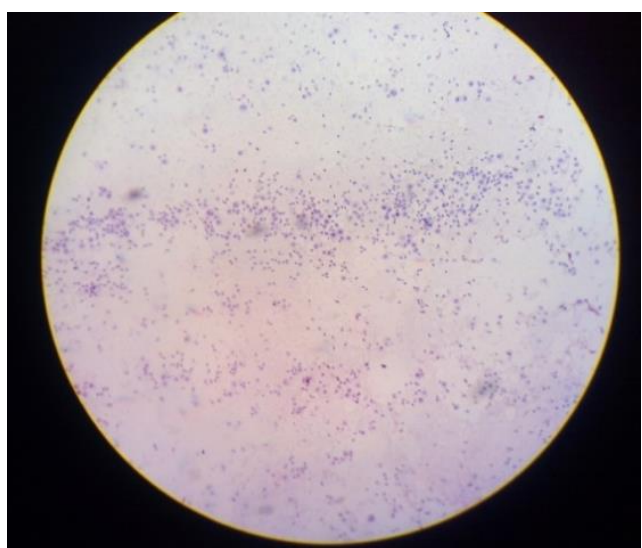

Fig-3a: 5 minutes fixation (PAP STAIN 10X)

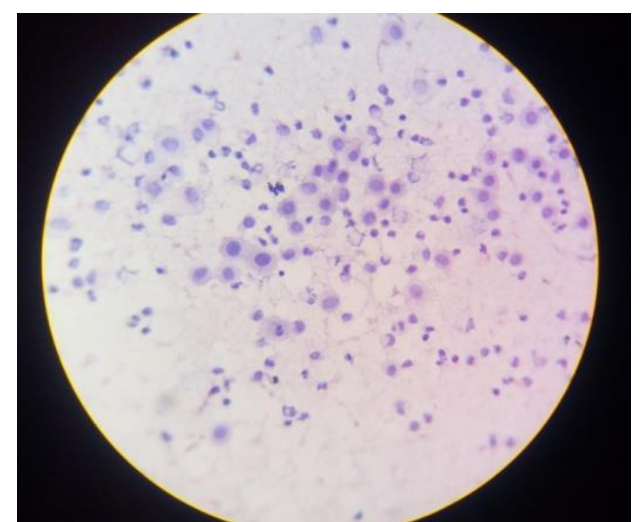

Fig-3b: 5minutes fixation (PAP STAIN 40X) 
Pleural fluid; smears show singly scattered reactive mesothelial cells and inflammatory cells in a eosinophilic back ground.

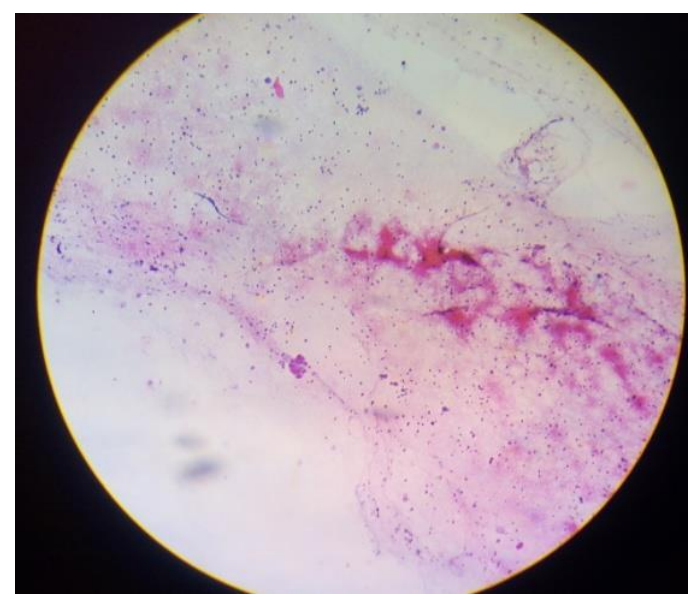

Fig-4a: >30 minutes fixation (PAP STAIN10X)

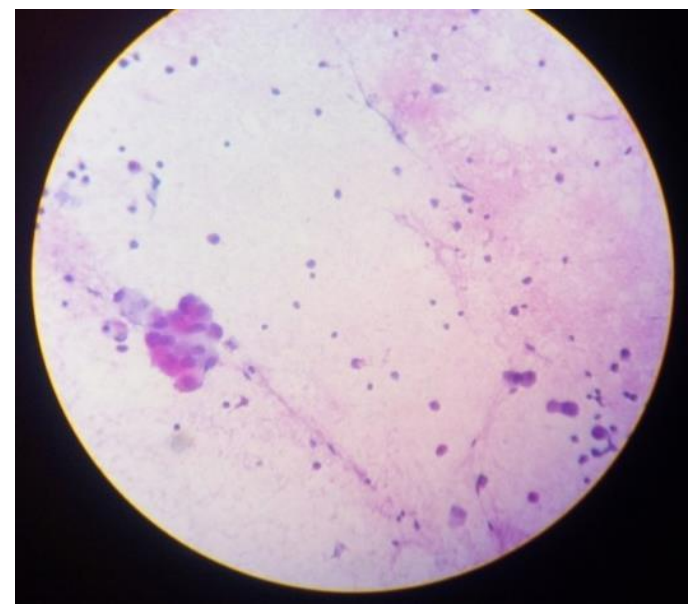

Fig-4b: >30 minutes fixation (PAP STAIN 40X)

Ascitic fluid: Smears show few reactive mesothelial cells a, inflammatory cells and RBCs in a eosinophilic background

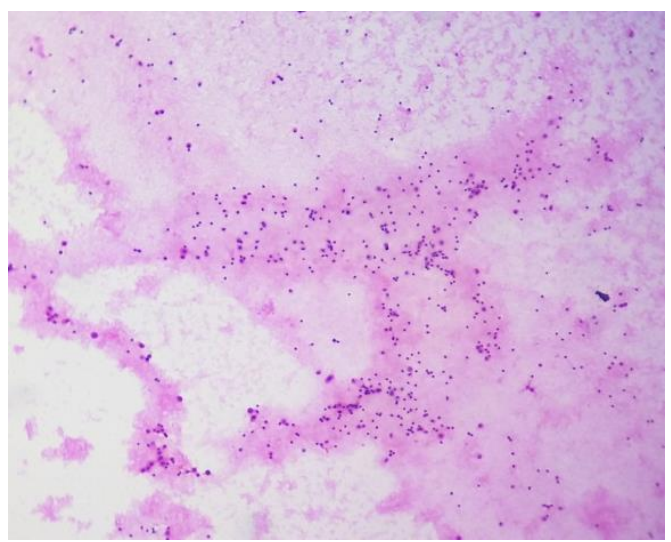

Fig 5a: >30 minutes fixation (H\&E 10X)
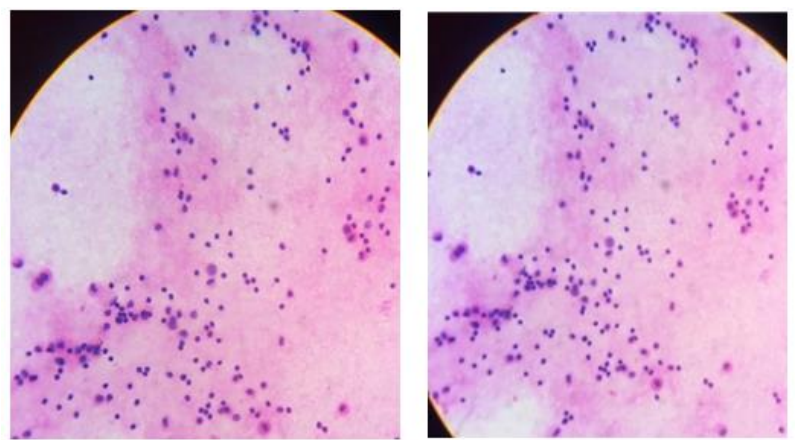

Fig-5 b \& c: >30 minutes fixation (H\&E 40X)

Pleural fluid; smears show small clusters and singly scattered reactive mesothelial cells and inflammatory cells in a eosinophilic back ground.

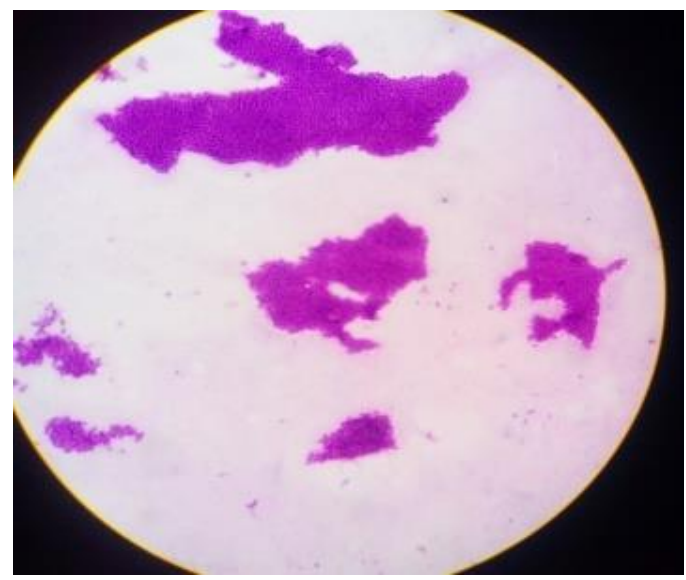

Fig-6a: 5 minutes fixation (H\&E 10X)

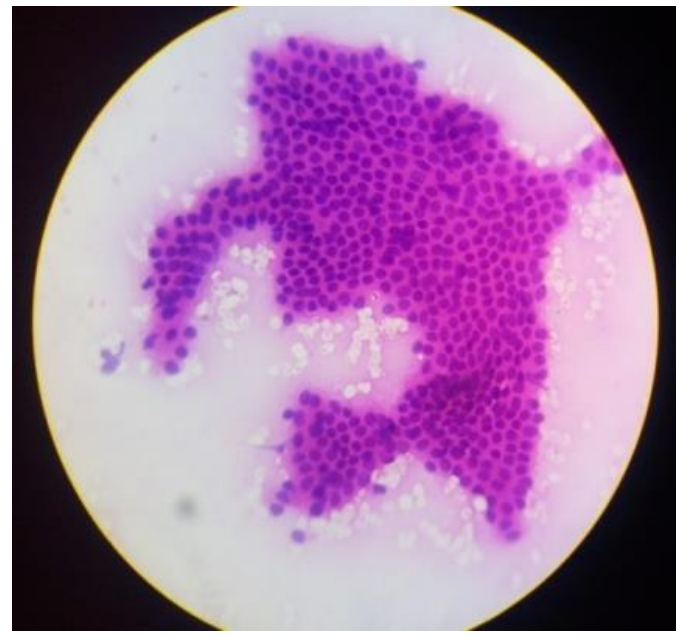

Fig-6b: 5 minutes fixation $(\mathrm{H} \& \mathrm{E} 40 \mathrm{X})$

FNAC: Fibroadenoma breast; Smears show cohesive clusters and monolayered sheets of benign ductal epithelial cells and few bare nuclei in hemorrhagic back ground. 


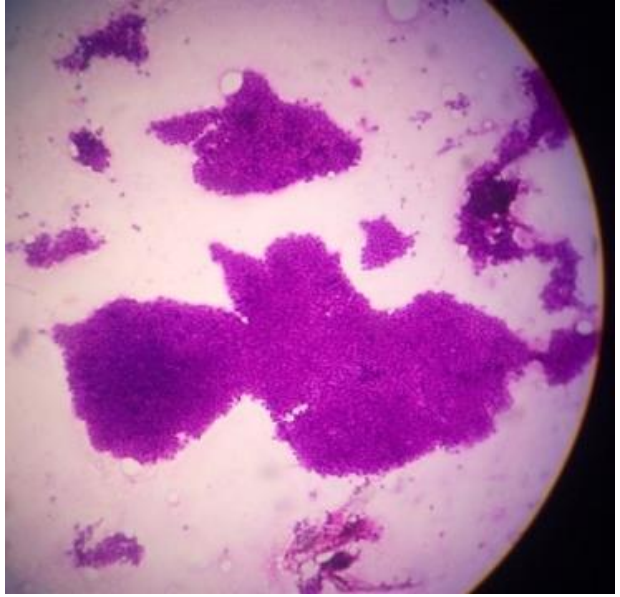

Fig-7a: >30 minutes fixation (H\&E 10X)

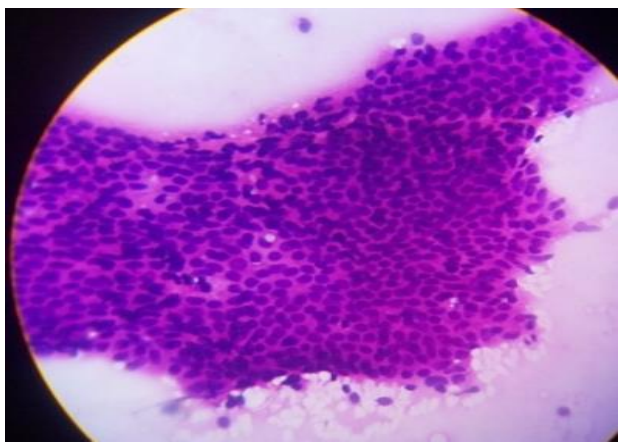

Fig-7b: (H\&E 40X)

FNAC: Fibroadenoma breast; Smears show cohesive clusters and monolayered sheets of benign ductal epithelial cells and few bare nuclei in hemorrhagic back ground.

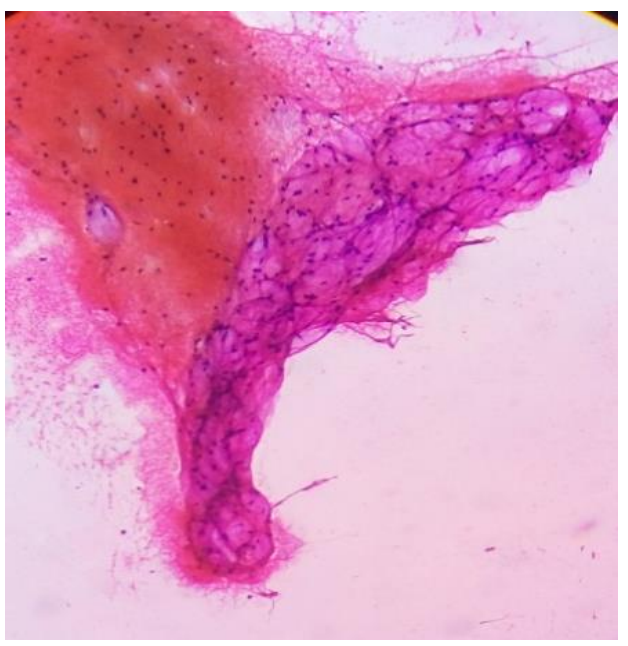

Fig-8a: 5 minutes fixation (H\&E 10X)

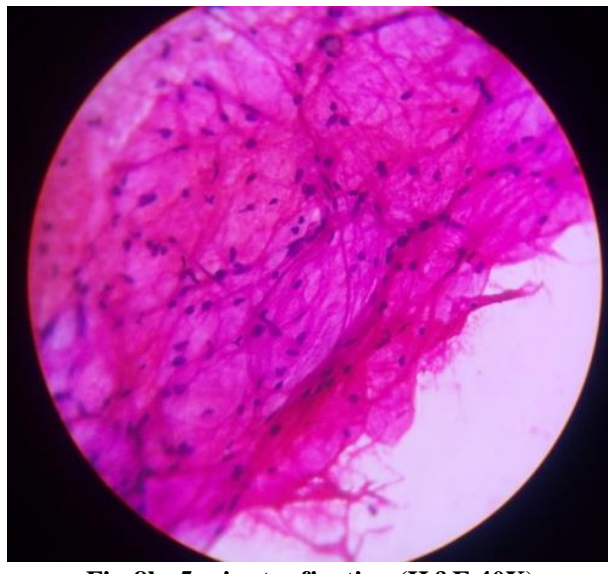

Fig-8b: 5 minutes fixation (H\&E 40X)

FNAC, Lipoma: smears show lobules of mature adipocytes and haemorrhage.

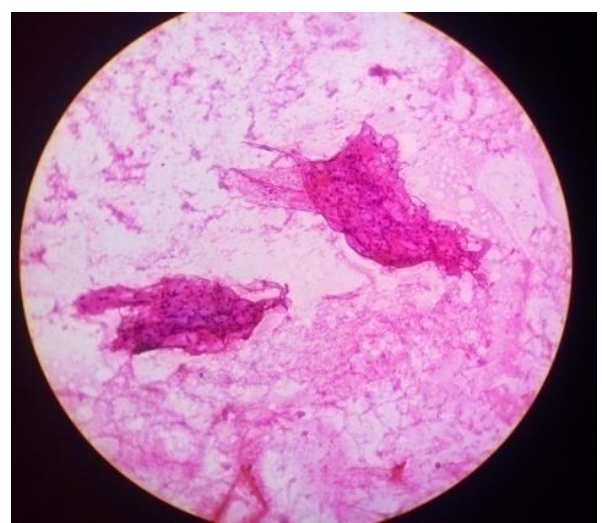

Fig-9a: >30 minutes fixation $(\mathrm{H} \& \mathrm{E}$ 10X)

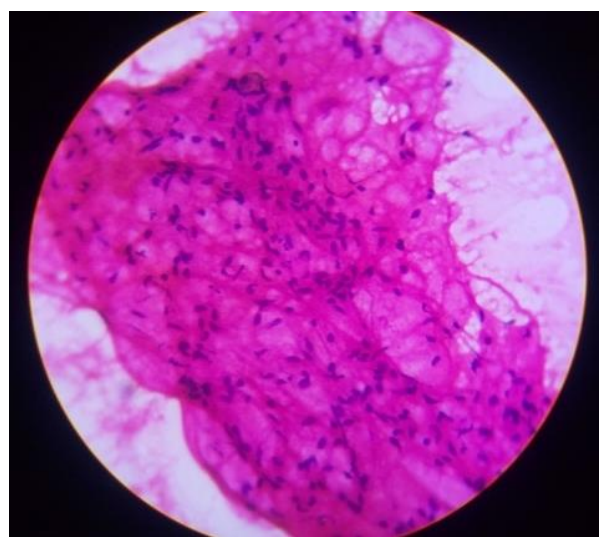

Fig-9b: >30 minutes fixation (H\&E 40X)

FNAC, Lipoma: smears show lobules of mature adipocytes and haemorrhage.

\section{DISCUSSION}

The accurate diagnosis of cytoloical smears are based on how fluid samples are fixed. The purpose of cytologic fixatives is to maintain the cytomorphologic characteristics of the cell [5]. Proper fixation of FNAC smears and body fluids is essential when it comes to laboratory for analysing cytological changes. If smears are allowed to dry prior to fixation, marked distortion of cells occurs [6]. So rapid fixation 
of smears is necessary to preserve cytologic detail of cells

It is important that all cytological smear preparations should fixed immediately before drying of the material occurs and to prevent cellular distortion [1]. The fixative should be capable of penetrating rapidly with good preservation of cell morphology. The fixatives used in cytological smears are $80 \%$ isopropyl alcohol, an excellent fixative and gives good nucleus and cytoplasmic morphology $.95 \%$ ethyl alcohol and $3 \%$ glacial acetic acid. Glacial acetic acid increases the nucleoprotein fixing properties. Ether alcohol is most widely used fixative and methanol also can be used as a cytological fixative $[1,7]$

The cytological stains used in cytology laboratory are papanicolaous stain, romanowsky stains like Giemsa, wrights and leishmans stain, and hematoxylin and eosin.

The properties of fixative are penetrate cells rapidly, minimize cell shrinkage, maintain morphologic integrity, inactivate autolytic enzymes, replace cellular water, allow permeability of dyes across cell boundaries, permit cell adhesion to a glass surface, matched to the subsequent staining method used, bactericidal, reproducible, represent a permanent cellular record.

Types of fixation methods [1]:

1. Wet fixation ;immediate submersion of wet cell samples into a fixative solution

2. Wet fixation with subsequent air drying

3. Spray fixative; immediate fixation of wet cell sample with a spray fixative.

Routinely the smears should remain in fixative for a minimum of 20-30 minutes prior to staining and also some smears require a minimum of 1 hour for complete fixation.

Till now there are no studies in fixation of smears by 5 minutes. Hopefully our study is the first one using this method

\section{CONCLUSION}

The cytoplasmic and background staining quality is good in both fixation timings. There is little slight variation in nuclear features in 5 minutes are satisfactory or Good and in more than 20-30 minutes are good and excellent.

By rapid fixation the adequacy of the smears can be analysed quickly. If the smears are scanty cellular or inadequate or insufficient material to report, we can repeat the procedure after analysis of the slides. So we can reduce the waiting time and save the economy of the patient. We can report more slides and the turnaround time are also reduced.

ACKNOWLEDGEMENTS: Am thankful to our technician vinod and BSc MLT interns

\section{Funding: Nil \\ Conflict of Interests: No}

\section{REFERENCES}

1. Hand Book of Medical laboratory Technology, Edited by Robert H. Carman, M.D. 533-535.

2. Nayak, R. (2017). Histopathology techniques and its management. JP Medical Ltd.

3. Hand book of Histopathological and Histochemical Technoues. $3^{\text {rd }}$ edition 1974. C.F.A Culling. Butter worth and Co publishers Ltd. Cytological fixatives. 50-53.

4. Deshpande, A. K., \& Bhaskaran, S. (2015). Comparative study of body fluid cytology using Cytospin-II and ordinary centrifuge. Journal of Evolution Of Medical And Dental SciencesJemds, 4(40), 6904-6910.

5. Koss. (2005). A book of diagnostic cytology and its histopathology basis volume $25^{\text {th }}$ edition Lippincott Williams and Wilkins chapter 31, cytopreparatory techniques. 1570-1634

6. Benjammin, Cummings - introduction to body fluids.

7. Kumarasinghe, M. P., Constantine, S. R., \& Hemamali, R. L. (1997). Methanol as an alternative fixative for cytological smears. The Malaysian journal of pathology, 19(2), 137-140. 